\title{
Transcriptome analysis of Pueraria candollei var. mirifica for gene discovery in the biosyntheses of isoflavones and miroestrol
}

\author{
Nithiwat Suntichaikamolkul', Kittitya Tantisuwanichkul ${ }^{1}$, Pinidphon Prombutara ${ }^{2}$, Khwanlada Kobtrakul ${ }^{3}$, \\ Julie Zumsteg ${ }^{4}$, Siriporn Wannachart ${ }^{5}$, Hubert Schaller ${ }^{4}$, Mami Yamazaki ${ }^{6}$, Kazuki Saito $^{6}$, Wanchai De-eknamkul ${ }^{7}$, \\ Sornkanok Vimolmangkang ${ }^{7}$ and Supaart Sirikantaramas ${ }^{1,2^{*}}$
}

\begin{abstract}
Background: Pueraria candollei var. mirifica, a Thai medicinal plant used traditionally as a rejuvenating herb, is known as a rich source of phytoestrogens, including isoflavonoids and the highly estrogenic miroestrol and deoxymiroestrol. Although these active constituents in P. candollei var. mirifica have been known for some time, actual knowledge regarding their biosynthetic genes remains unknown.

Results: Miroestrol biosynthesis was reconsidered and the most plausible mechanism starting from the isoflavonoid daidzein was proposed. A de novo transcriptome analysis was conducted using combined $P$. candollei var. mirifica tissues of young leaves, mature leaves, tuberous cortices, and cortex-excised tubers. A total of 166,923 contigs was assembled for functional annotation using protein databases and as a library for identification of genes that are potentially involved in the biosynthesis of isoflavonoids and miroestrol. Twenty-one differentially expressed genes from four separate libraries were identified as candidates involved in these biosynthetic pathways, and their respective expressions were validated by quantitative real-time reverse transcription polymerase chain reaction. Notably, isoflavonoid and miroestrol profiling generated by LC-MS/MS was positively correlated with expression levels of isoflavonoid biosynthetic genes across the four types of tissues. Moreover, we identified R2R3 MYB transcription factors that may be involved in the regulation of isoflavonoid biosynthesis in P. candollei var. mirifica. To confirm the function of a key-isoflavone biosynthetic gene, $P$. candollei var. mirifica isoflavone synthase identified in our library was transiently co-expressed with an Arabidopsis MYB12 transcription factor (AtMYB12) in Nicotiana benthamiana leaves. Remarkably, the combined expression of these proteins led to the production of the isoflavone genistein.
\end{abstract}

Conclusions: Our results provide compelling evidence regarding the integration of transcriptome and metabolome as a powerful tool for identifying biosynthetic genes and transcription factors possibly involved in the isoflavonoid and miroestrol biosyntheses in P. candollei var. mirifica.

Keywords: Pueraria candollei var. mirifica, White Kwao Krua, Miroestrol, Isoflavones, Transcriptome

\footnotetext{
* Correspondence: Supaart.S@chula.ac.th

${ }^{1}$ Department of Biochemistry, Faculty of Science, Chulalongkorn University,

Bangkok, Thailand

${ }^{2}$ Omics Sciences and Bioinformatics Center, Chulalongkorn University,

Bangkok, Thailand

Full list of author information is available at the end of the article
}

(c) The Author(s). 2019 Open Access This article is distributed under the terms of the Creative Commons Attribution 4.0 International License (http://creativecommons.org/licenses/by/4.0/), which permits unrestricted use, distribution, and reproduction in any medium, provided you give appropriate credit to the original author(s) and the source, provide a link to the Creative Commons license, and indicate if changes were made. The Creative Commons Public Domain Dedication waiver (http://creativecommons.org/publicdomain/zero/1.0/) applies to the data made available in this article, unless otherwise stated. 


\section{Background}

White Kwao Krua (Pueraria candollei var. mirifica, hereafter shortened to P. mirifica, shown in Additional file 1: Figure S1), has been extensively used in Thai traditional medicine as a rejuvenating herb because of its numerous phytoestrogenic constituents [1]. Phytoestrogens are plant-derived compounds that structurally or functionally mimic mammalian estrogen, and they have been applied to treat different forms of cancer, heart disease, menopausal symptoms, and osteoporosis [2]. Considering the numerous effects of phytoestrogens on human health, $P$. mirifica may be a promising candidate for treating various diseases and for developing novel medicinal products.

Three major types of phytoestrogens occur in $P$. mirifica: isoflavones, coumestans, and chromenes [3-5]. Isoflavones are an important type of phytoestrogens, which are biosynthesized via the phenylpropanoid pathway and occur predominantly in leguminous plants [6]. Seven isoflavones that have been identified in $P$. mirifica tubers: puerarin, daidzin, genistin, daidzein, genistein, kwakhurin, and mirificin [3, 5]. Four coumestans that also occur in the tuber of this plant are coumestrol, mirificoumestan, mirificoumestan hydrate, and mirificoumestan [7]. The chemical structure of chromenes has received considerable attention because of their low toxicity and broad pharmacological application as anticancer, antimicrobial, and anti-inflammatory agents [8]. Miroestrol and its precursor deoxymiroestrol are typically accumulated at very low levels [9], however, these compounds are the predominant chromenes in the tuberous cortex of $P$. mirifica [10], and both compounds exhibit considerably highest estrogenic activity [11]. Since both chromenes have not been reported in other plant species, their biosynthesis is likely unique to $P$. mirifica. Interestingly, although miroestrol has been identified almost six decades ago, its biosynthetic pathway and the enzymes involved are still unknown.

Regarding biosynthesis of phytoestrogens, the early steps of isoflavonoid and flavonoid are generally similar, starting with phenylalanine ammonia lyase removing amides from the first substrate, phenylalanine, to produce cinnamic acid that is hydroxylated by cinnamate 4hydroxylase to produce $p$-coumarate. The enzyme 4coumarate-CoA ligase then activates $p$-coumarate by attaching a co-enzyme A (coA), and subsequently, chalcone synthase (CHS) binds $p$-coumaroyl-CoA to three molecules of malonyl-CoA to form a chalcone skeleton. Chalcone can be converted to flavanone by chalcone isomerase. Liquiritigenin is a substrate for both the flavonoid and the isoflavonoid pathway. Isoflavonoids are generally synthesized from common intermediates (either liquiritigenin or naringenin) within the recognized flavonoid biosynthetic pathway by aryl migration, which is catalyzed by isoflavone synthase
(IFS). This pathway leads to the formation of an intermediate product, 2-hydroxyisoflavanone, which is then dehydrated to daidzein through catalysis by 2hydroxyisoflavanone dehydratase (Fig. 1) [12]. CHS and IFS have been identified in $P$. mirifica several years ago $[13,14]$, however, their enzymatic functions have not been elucidated. Udomsuk et al. [15] suggested that miroestrol biosynthesis may share a common pathway with isoflavonoid biosynthesis due to their similar backbone structure. So far, no biosynthetic genes or enzymes involved in miroestrol biosynthesis have been reported, thus also the transcription factors responsible for regulating the expression of these biosynthetic genes remain to be identified. V-myb myeloblastosis viral oncogene homolog transcription factors (MYB TFs), which are the largest plant transcription factor family, have been reported to possess key functions in regulating the synthesis of phenylpropanoid-derived compounds in plants [16]. These proteins have attracted substantial interest regarding phytoestrogen biosynthesis in plants.

Transcriptomes produced from high-throughput sequencing of various plants are a potential source for identifying genes involved in the biosynthesis of different secondary metabolites. The transcriptome of Pueraria lobata, a species closely related to $P$. mirifica, has been published previously $[17,18]$. Although these two plants produce similar types of isoflavones, miroestrol occurs only in $P$. mirifica. In the afore-mentioned studies, $P$. lobata genes that encode core isoflavone biosynthetic enzymes were identified, and their expression levels in various tissues were examined.

In the current study, we high-throughput sequenced $P$. mirifica to produce transcriptome libraries of young leaves, mature leaves, cortex-excised tubers, and tuberous cortices, and we de novo assembled the transcriptomes to characterize the biosynthetic pathway of miroestrol. Additionally, MYB TFs involved in isoflavonoid biosynthesis were also identified. This integrative approach of using transcriptomics and metabolomics provides new insights for the prediction and identification of putative biosynthetic genes and transcription factors that are potentially involved in $P$. mirifica isoflavonoid and miroestrol biosynthetic pathways.

\section{Results}

\section{De novo transcriptome assembly of $P$. mirifica}

To obtain nucleotide sequences of expressed genes in various tissues of $P$. mirifica, we constructed a cDNA library from pooled tissues including, young leaves, mature leaves, cortex-excised tubers, and tuberous cortices (Fig. 2a-d). The library was processed using an Illumina Hiseq 2000 platform, yielding approximately $8.2 \mathrm{G}$ base pairs and a total of 7,386,137,640 clean nucleotides (nt). The de novo assembly resulted in 166,923 contigs (62, 


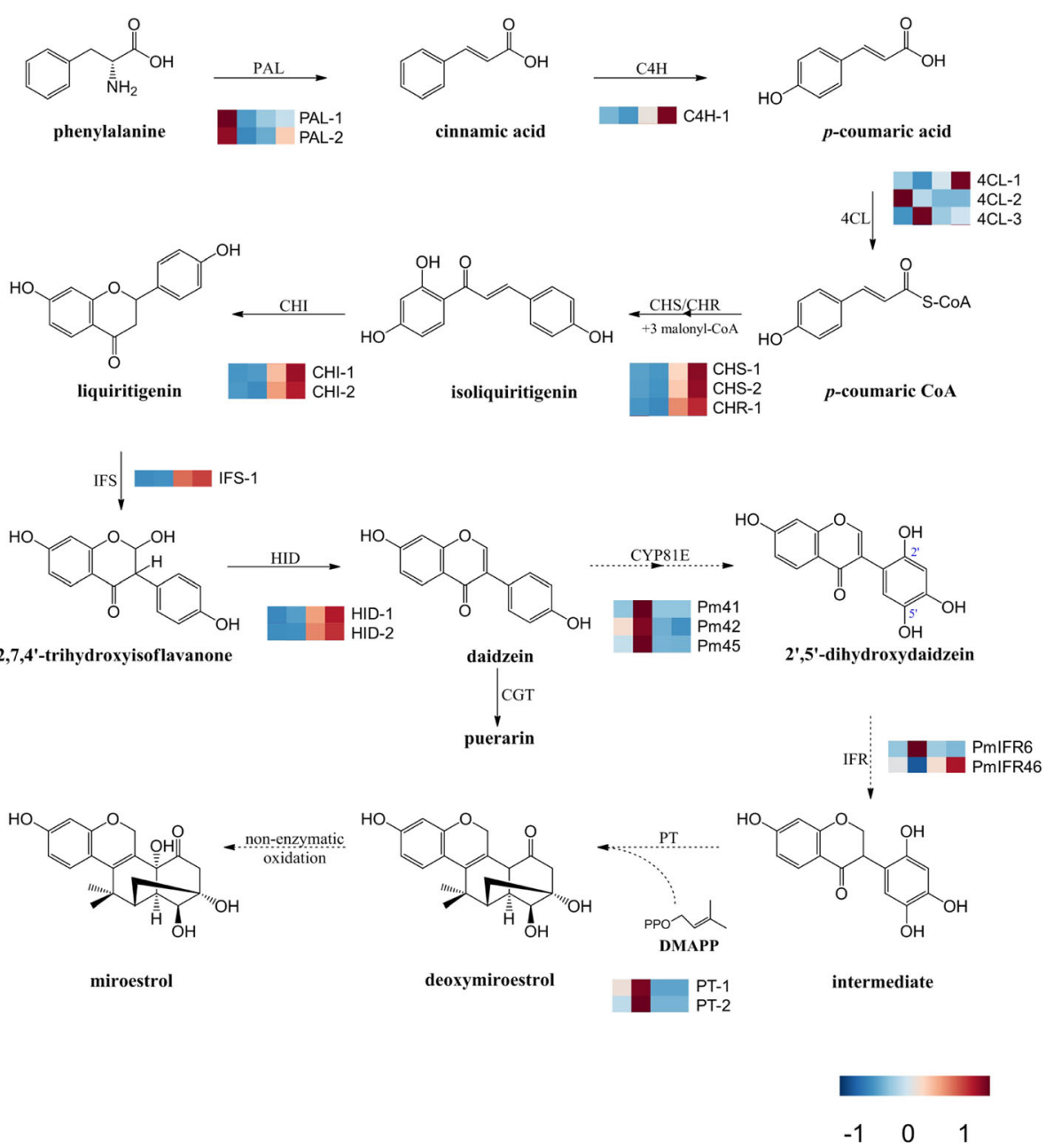

Fig. 1 Proposed miroestrol biosynthetic pathway in P. mirifica. The heatmap following each gene name indicates the relative differential expression of genes in young leaves, mature leaves, tubers without cortices, and cortices of $P$. mirifica, respectively. Enzyme abbreviation: PAL; Phenylalanine ammonialyase, C4H; Cinnamate 4-monooxygenase, 4CL; Coumarate-CoA ligase, CHS; Chalcone synthase, CHI; Chalcone isomerase, IFS; 2-hydroxyisoflavone synthase, HID; 2-hydroxyisoflavone dehydratase, CGT; C-glycosyltransferase, CYP81E; Cytochrome P450 subfamily 81E, IFR; Isoflavone reductase, PT; Prenyltransferase. Although the assembled unigenes are hypothetical candidate genes, a comparative transcriptome analysis using $P$. lobata is a promising way to study and identify species-specific and evolutionary conserved pathways involved in the highly complex biosynthesis of miroestrol

$567,517 \mathrm{nt})$ and 104,283 unigenes $(81,810,584 \mathrm{nt})$, the mean length/N50 for contigs and unigenes was 375/734 nt and 785/1558 nt, respectively. To assess the completeness of transcriptome data, we performed a BUSCO analysis compared to the 2121 single-copy orthologs of the eudicot lineage. The de novo transcriptome assembly was complete to $87.4,6.9 \%$ of contigs were fragmented, and $5.7 \%$ of the transcriptome was missing (Additional file 7: Table S1). In addition, the assembly produced here was compared to that of other leguminous plants (summarized in Table 1).

\section{Functional annotation and classification of protein}

Functional annotation of the assembled transcripts provides insights in potential metabolic functions and biological processes within an organism. The functional annotation of $P$. mirifica assembled transcripts was performed based on similarities with proteins or transcripts according to information that is available in various public databases. The statistics of functional annotation are summarized in Additional file 8: Table S2. Aligned unigenes showed significant homologies using the National Center for Biotechnology Information (NCBI) non-redundant protein database. Based on the BLAST similarity distribution, over $68 \%$ of unigenes exhibited similarities greater than $80 \%$. A top-hit species distribution analysis showed unigenes with BLAST hits sharing high sequence similarities with Glycine $\max (85.3 \%)$, Medicago truncatula (6.2\%), and Vitis vinifera (1.6\%).

The clusters of orthologous groups (COG) indicated the functional classification of each unigene at the cellular level. In total, 1091 unigenes were predicted to be involved 


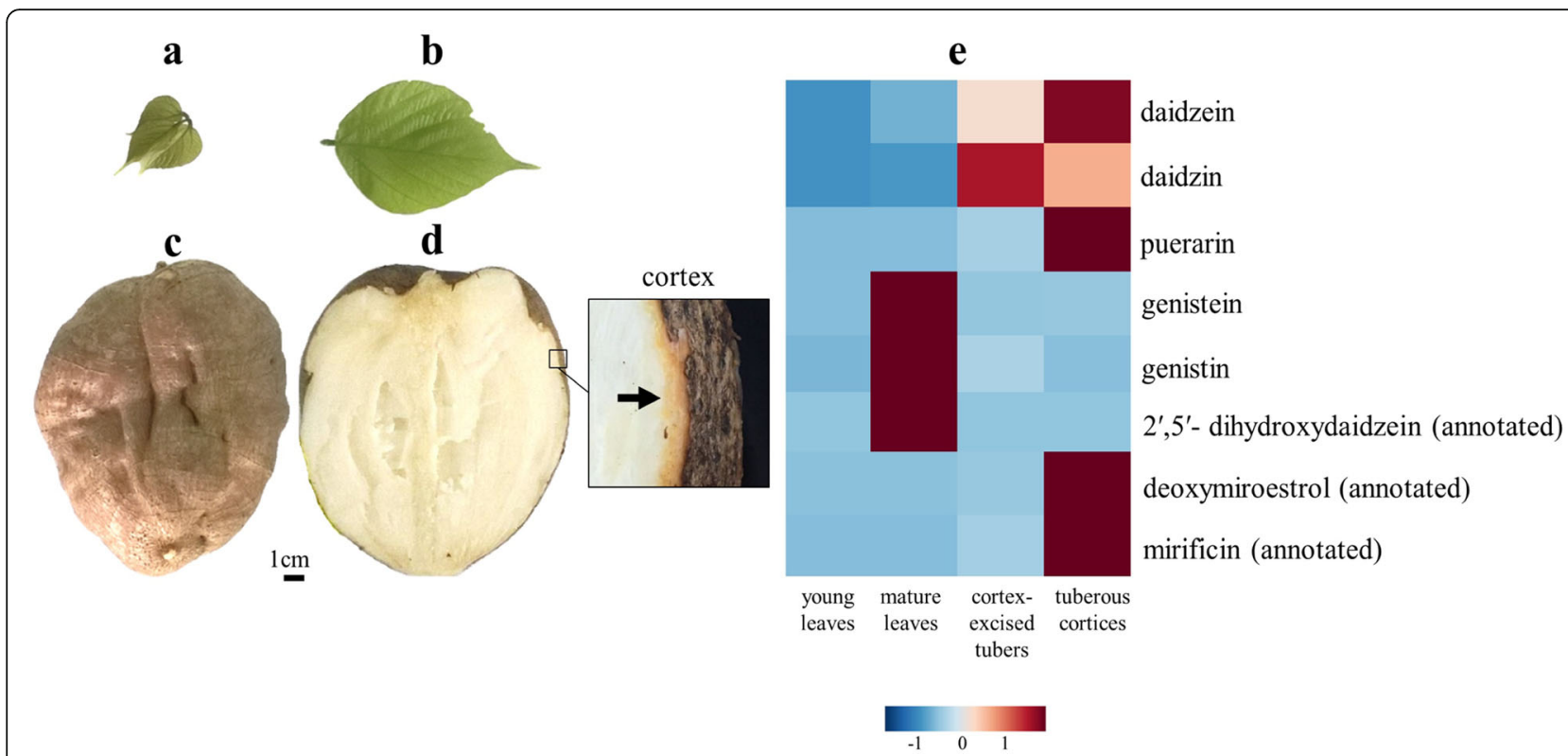

Fig. 2 Young leaves (a), mature leaves (b) and tuber (c) morphology of P. mirifica cultivar SARDI19 used for transcriptome assembly, RNA-Seq, qRT-PCR, and HPLC-QTOF-MS/MS analysis (left). Cross section of three-year old whole tuber (d). Accumulation of isoflavonoids and chromenes in four different tissues of $P$. mirifica as measured with HPLC-QTOF-MS/MS (e). The analysis was performed with three biological replicates

in secondary metabolite biosynthesis (Additional file 2: Figure S2). Gene ontology (GO), which classifies standardized gene function, is useful for annotating gene functions and gene products in various organisms. GO is based on three major dependent factor categories: biological processes, molecular functions, and cellular components. The 37,058 unigenes yielded a corresponding GO term that can be further classified into 56 sub-categories: 23 categories related to biological processes, 17 to cellular components, and 16 to molecular functions. Additional file 3: Figure S3 shows the substantial number of transcripts related to cellular components and metabolic processes. The remaining 67,225 unigenes produced no BLAST hits. These unannotated unigenes may be uncharacterized genes or assembled sequences that were too short to produce hits.

The Kyoto Encyclopedia of Genes and Genomes (KEGG) is a comprehensive database for identifying biological pathways and for functional annotation of gene products. Pathway-based annotation helps produce an overview of the different metabolic processes that are active within an organism, and it helps improve our

Table 1 Comparison of $P$. mirifica de novo assembly to other leguminous plants

\begin{tabular}{|c|c|c|c|c|c|}
\hline Description & P. mirifica & P. lobata [18] & Ammopiptanthus mongolicus [19] & Millettia pinnata [20] & Medicago sativa [21] \\
\hline Total Clean Reads (Mb) & 82 & 73 & 67 & 80 & 29 \\
\hline Total Clean Nucleotides (Mb) & 7386 & 6974 & 6056 & 7219 & 5643 \\
\hline \multicolumn{6}{|l|}{ Contig } \\
\hline Total Number (kb) & 167 & 336 & 149 & 109 & 81 \\
\hline Total Length (Mb) & 63 & 390 & 51 & 40 & 71 \\
\hline Mean Length (nt) & 375 & 1162 & 345 & 365 & 873 \\
\hline N50 (nt) & 734 & 1988 & 619 & 682 & 1323 \\
\hline \multicolumn{6}{|l|}{ Unigene } \\
\hline Total Number (kb) & 104 & 164 & 85 & 54 & 40 \\
\hline Total Length (Mb) & 82 & 112 & 57 & 42 & 33 \\
\hline Mean Length (nt) & 785 & 683 & 675 & 787 & 803 \\
\hline N50 (nt) & 1558 & 1153 & 1191 & 1204 & 1300 \\
\hline
\end{tabular}

Values shown in $\mathrm{Mb}$ and $\mathrm{Kb}$ are rounded. Mb Megabase, kb Kilobase, $n t$ Nucleotide. 
understanding of biological functions of unigenes. All unigenes were analyzed using the KEGG pathway with an $e$-value cutoff of $<10^{-5}$. We obtained 33,317 unigenes, 3997 of which were related to biosynthesis of secondary metabolites and 8103 to general metabolic pathways. The top-five ranking pathways were plant hormone signal transduction (2061 unigenes), endocytosis (1254 unigenes), RNA transportation (1232 unigenes), glycerophospholipid metabolism (1131 unigenes), and purine metabolism (1113 unigenes). Regarding the crucial capacity of leguminous plants to accumulate functional flavonoids, 476 flavonoid biosynthetic unigenes and 167 isoflavonoid biosynthetic unigenes are shown in Additional file 4: Figure S4. These functional annotations were used for identifying genes involved in isoflavonoid biosynthesis in P. mirifica.

\section{Proposed miroestrol biosynthetic pathway, differential} accumulation of transcripts associated with isoflavonoids, and miroestrol biosynthesis

Miroestrol biosynthesis potentially shares a pathway with isoflavonoid biosynthesis [15]. We propose that daidzein, a common isoflavone aglycone, is hydroxylated by at least two cytochrome P450 enzymes at the $2^{\prime}$ and 3 ' carbon of the B-ring to produce 2',5'-dihydroxydaidzein; these enzyme may be members of the CYP81E subfamily which is known to use isoflavones as substrates [22-25]. Then, 2',5'-dihydroxydaidzein would be reduced by isoflavone reductase and subsequently would be prenylated by a prenyltransferase using dimethylallyl diphosphate as a co-substrate to produce deoxymiroestrol and miroestrol (Fig. 1).

Based on a functional annotation of each unigene found in the P. mirifica de novo transcriptome assembly and phylogenetic analyses, a total of 14 putative genes involved in isoflavone biosynthesis were predicted as follows: two $P A L$ genes (encoding phenylalanine ammonia lyase), one $\mathrm{C} 4 \mathrm{H}$ gene (encoding cinnamate 4-hydroxylase), three 4CL genes (encoding 4-coumarate-CoA ligase), two CHS genes (encoding chalcone synthase), one CHR gene (encoding chalcone reductase), two $\mathrm{CHI}$ genes (encoding chalcone isomerase), one IFS gene (encoding IFS), and two HID genes (encoding 2-hydroxyisoflavanone dehydratase). In addition, a total of seven putative genes were identified in our proposed miroestrol biosynthetic pathway: three CYP81E genes, two IFR genes (encoding isoflavone reductase), and two $P T$ genes (encoding prenyltransferase). To investigate gene expression levels of these unigenes across four tissues of $P$. mirifica, reads per kilobase million of transcripts were calculated from the RNA sequencing data generated using a NextSeq500 platform. An analysis of differentially expressed genes (DEG) on these unigenes (probability threshold $\mathrm{q}=0.9$ ) was visualized as a heat map based on our proposed miroestrol biosynthetic pathway
(Fig. 1). Their sequences and RPKM values are shown in Additional file 9: Table S3. Additionally, a total of 16 putative genes encoding UDP-glycosyltransferase that are potentially involved in this pathway were phylogenetically identified from the transcriptome data. Their DEGs across the four tissue types are shown as a heatmap in Additional file 5: Figure S5.

To validate the DEG analysis obtained from RNA sequencing, candidate unigenes were selected and analyzed based on their expression levels in the four tissue types using quantitative real-time reverse transcription polymerase chain reaction (qRT-PCR). As expected, expression levels of the selected unigenes were positively correlated with DEGs identified by RNA sequencing (Fig. 3). Indeed, most of the isoflavone biosynthetic genes were highly expressed in tuberous cortices, compared to young leaves, mature leaves, and cortex-excised tubers; however, PmCYP81Es, PmIFRs, and PmPT higher expressed in mature leaves than in the other tissues.

\section{Phytoestrogens and annotated constituents in P. mirifica}

High-performance liquid chromatography (HPLC) coupled to tandem mass spectroscopy (MS) is a powerful tool with high selectivity and sensitivity. Seven phytoestrogens were identified in $P$. mirifica by comparison with standard compounds based on their retention times and MS fragmentation patterns. These phytoestrogens included daidzein, daidzin, genistein, genistin, 2'-hydroxydaidzein, 3'-hydroxydaidzein, and puerarin (Table 2). Due to the lack of standard compounds, mirificin, 2',5'-dihydroxydaidzein, deoxymiroestrol, and miroestrol were predicted on the basis of mass accuracy ranges and MS fragmentation patterns searches conducted on the METLIN metabolomics database [26] or/and comparisons with published data [27]. The relative abundance of major phytoestrogens found in young leaves, mature leaves, cortexexcised tubers, and tuberous cortices of $P$. mirifica are shown as a heat map in Fig. 2e. Genistein, genistin, and annotated 2', 5'-dihydroxydaidzein were highly accumulated in mature leaves, whereas daidzein, puerarin, annotated mirificin, and annotated deoxymiroestrol were highly accumulated in tuberous cortices, compared to the other tissues. Although miroestrol was not detected in any tissue, its precursor (annotated deoxymiroestrol) was observed only in P. mirifica tuberous cortex, which is the main accumulation site of miroestrol [10].

\section{Isoflavone production in $N$. benthamiana leaves over- expressing $P$. mirifica isoflavone synthase}

Recently, $N$. benthamiana has been used to identify transient expression of several plant genes to confirm gene functions [28]. To demonstrate that our transcriptome libraries contained candidate genes involved in $P$. mirifica phytoestrogen biosynthesis, we cloned $P$. mirifica 


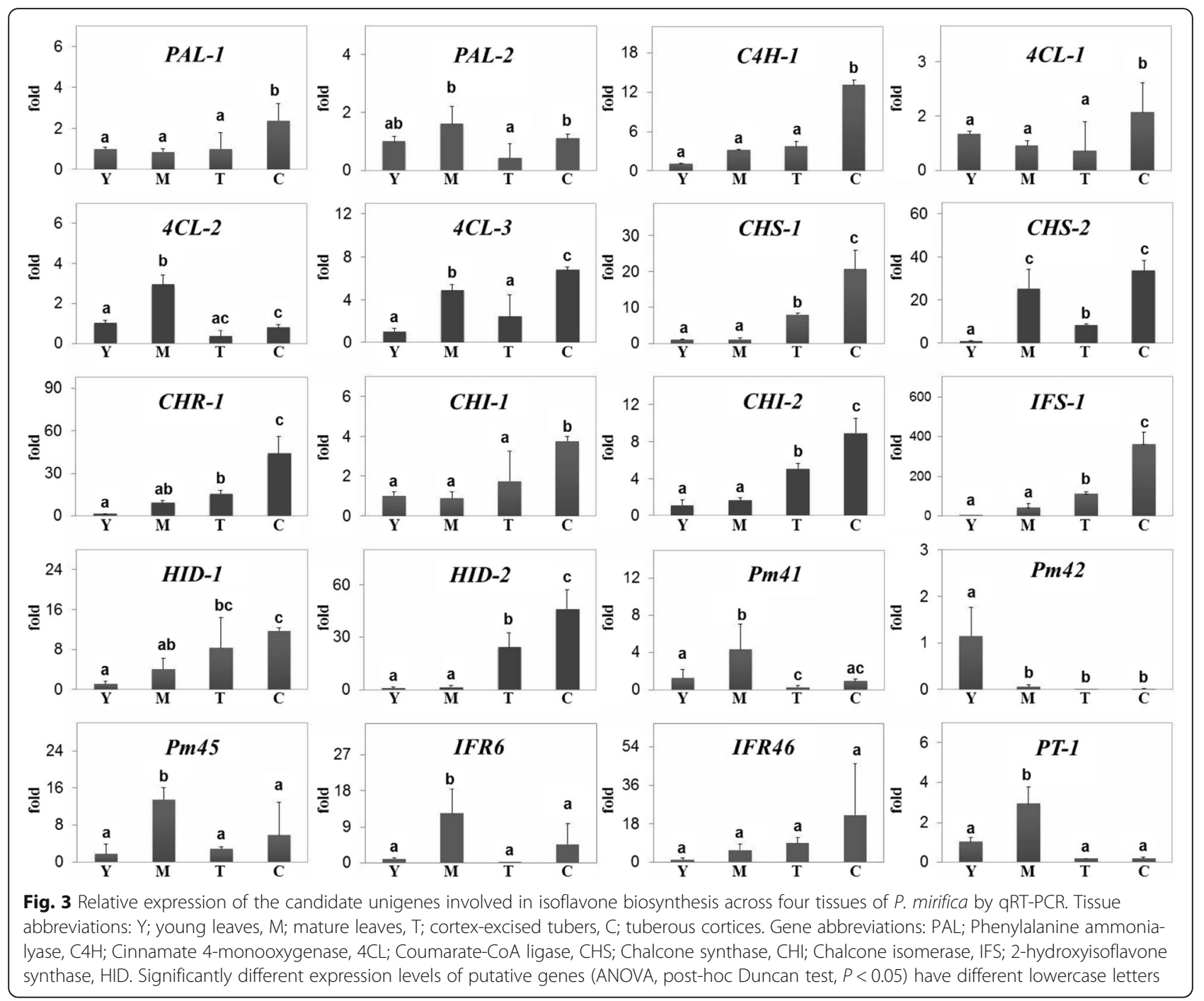

Table 2 Summary of the retention times and exact masses of identified compounds

\begin{tabular}{llll}
\hline Compound name & Chemical formula & Retention time (minute) & Exact mass \\
\hline mirificin & $\mathrm{C}_{26} \mathrm{H}_{28} \mathrm{O}_{13}$ & 10.07 & 548.15299 \\
daidzin & $\mathrm{C}_{21} \mathrm{H}_{20} \mathrm{O}_{9}$ & 10.16 & 416.11073 \\
puerarin & $\mathrm{C}_{21} \mathrm{H}_{20} \mathrm{O}_{9}$ & 10.40 & 416.11073 \\
genistin & $\mathrm{C}_{21} \mathrm{H}_{20} \mathrm{O}_{10}$ & 13.70 & 432.10565 \\
3'-hydroxydaidzein & $\mathrm{C}_{15} \mathrm{H}_{10} \mathrm{O}_{5}$ & 14.75 & 270.05282 \\
deoxymiroestrol & $\mathrm{C}_{20} \mathrm{H}_{22} \mathrm{O}_{5}$ & 15.03 & 342.14672 \\
2'-hydroxydaidzein & $\mathrm{C}_{15} \mathrm{H}_{10} \mathrm{O}_{5}$ & 15.10 & 270.05282 \\
daidzein & $\mathrm{C}_{15} \mathrm{H}_{10} \mathrm{O}_{4}$ & 16.33 & 254.05791 \\
2',5'-dihydroxydaidzein & $\mathrm{C}_{15} \mathrm{H}_{10} \mathrm{O}_{5}$ & 16.95 & 286.04774 \\
genistein & $\mathrm{C}_{15} \mathrm{H}_{10} \mathrm{O}_{5}$ & 18.78 & 270.05282 \\
\hline
\end{tabular}


isoflavone synthase (PmIFS) and conducted a functional characterization using transient (co-)expression in $N$. benthamiana, which does not produce any isoflavones. Transient expression of green fluorescent protein in fiveweek-old $N$. benthamiana leaves was used as a negative control. Co-expression of PmIFS and Arabidopsis R2R3 MYB12 transcription factor (AtMYB12), a regulator enhancing metabolic flux to flavonoid biosynthesis [29], generated two novel major peaks that were identified as genistein (Additional file 6: Figure S6), suggesting that PmIFS is involved in isoflavone biosynthesis.

\section{Identification of MYB transcription factors involved in isoflavone biosynthesis}

We identified 85 putative genes encoding MYB transcription factors in the P. mirifica transcriptome. All putative $P$. mirifica MYB transcription factors (PmMYB) were aligned to known MYB transcription factors of other plants such as Arabidopsis thaliana, Glycine max, and other species, and a phylogenetic tree was produced from this alignment (Fig. 4). Based on these analyses, we found six candidate PmMYBs that are potentially involved in the regulation of isoflavonoid biosynthesis. PmMYB18 was closely related to GmMYB29, which activates expression of IFS and CHS in soybean [30]. PmMYB23 and PmMYB24 clustered with GmMYB12B2, which regulates expression of $\mathrm{CHS}$ in soybean [31, 32]. PmMYB75, PmMYB76, and PmMYB77 clustered with GmMYB176, which is also involved in controlling $C H S$ expression and isoflavonoid synthesis in soybean [33, 34]. Furthermore, also PmMYB24 and PmMYB77 were highly expressed in mature leaves, whereas $P m M Y B 18$, PmMYB23, PmMYB75, and PmMYB76 were highly expressed in tuberous cortices (Fig. 5).

\section{Discussion}

De novo transcriptome assembly is a useful method for gathering comprehensive information on genetic resources without the need for whole genome sequencing. In addition, this technique facilitates discovery of novel genes, molecular markers, and tissue-specific expression patterns. In the absence of comprehensive genomic data of $P$. mirifica, RNA sequencing was used to explore the P. mirifica transcriptome. Although the Illumina HiSeq platform has various advantages over other methods in large-scale transcriptomics research, the output data requires more time for processing and analyses. The Illumina NextSeq platform was thus designed as a faster and easier operating benchtop device to reduce costs and data processing time. Overall error rates $(<1 \%)$ are comparable across these two platforms [35]. Here, we employed an Illumina HiSeq 2000 platform to produce a transcriptome library from four tissues, and an Illumina NextSeq 500 platform to determine expression patterns of genes in each transcriptome library produced from four tissues of $P$. mirifica. The BUSCO analysis confirmed that $94.3 \%$ of the complete and fragmented sequences were included in the assembly (Additional file 7: Table S1), suggesting that the vast majority of orthologs were covered by our de novo transcriptome assembly. We produced a large dataset of unigenes from the de novo transcriptome assembly generated on the HiSeq platform. The average GC content of those unigenes was $44.56 \%$. In eukaryotes, GC content averages approximately $20-60 \%$ [36]. The observed P. mirifica GC content was within this range, although slightly above those in P. lobata (39.9\%) [17] and Medicago truncatula (40\%) [37] but similar to that in Glycine max (43\%) [37]. Furthermore, most of the annotated unigenes produced matches in the Glycine max protein database, indicating that the respective functions are conserved in these two species. Although $P$. lobata is considered closely related to $P$. mirifica, only $0.12 \%$ of the annotated unigenes matched $P$. mirifica. This is due to the limited number of full-length sequences of $P$. lobata available in the protein database, and the currently available $P$. lobata NCBI reference transcriptome is raw sequencing data $[17,18]$. In addition, gene or protein names, descriptions of COG and GO terms, and possible metabolic pathways were also annotated. Notably, all candidate genes generated from the HiSeq platform data were found in the four libraries generated from the NextSeq platform data. The respective expression levels across the four tissues of $P$. mirifica can be used to reduce the number of candidates for further functional characterization.

Isoflavones are a class of flavonoids that are produced almost exclusively in leguminous plants [6]. In the $P$. mirifica transcriptome dataset, 21 putative genes involved in isoflavonoid biosynthesis were identified. The DEG values of these unigenes that were validated by qRT-PCR, exhibited high expression levels in tuberous cortices compared to those in the other three tissues. Consequently, almost all isoflavones, such as puerarin and daidzin, also showed the highest accumulation in the tuberous cortices. When comparing $P$. mirifica and $P$. lobata, gene expression and the isoflavone accumulation profile in each tissue were similar [17, 18], apart from the lack of IFS expression and isoflavonoid accumulation in the leaves of $P$. lobata [17]. This observation suggests a divergent evolution pattern in these two closely related species which occur in different regions (P. lobata in temperate zones and P. mirifica in tropical zones). It is possible that $P$. mirifica has evolved a mechanism to accumulate isoflavonoids in leaves and miroestrol in tuberous cortices as a means of defense. To assess whether our transcriptome data contained candidate genes, we cloned PmIFS into $N$. benthamiana, which does not naturally produced isoflavonoids, for transient co-expression of PmIFS and AtMYB12, an 


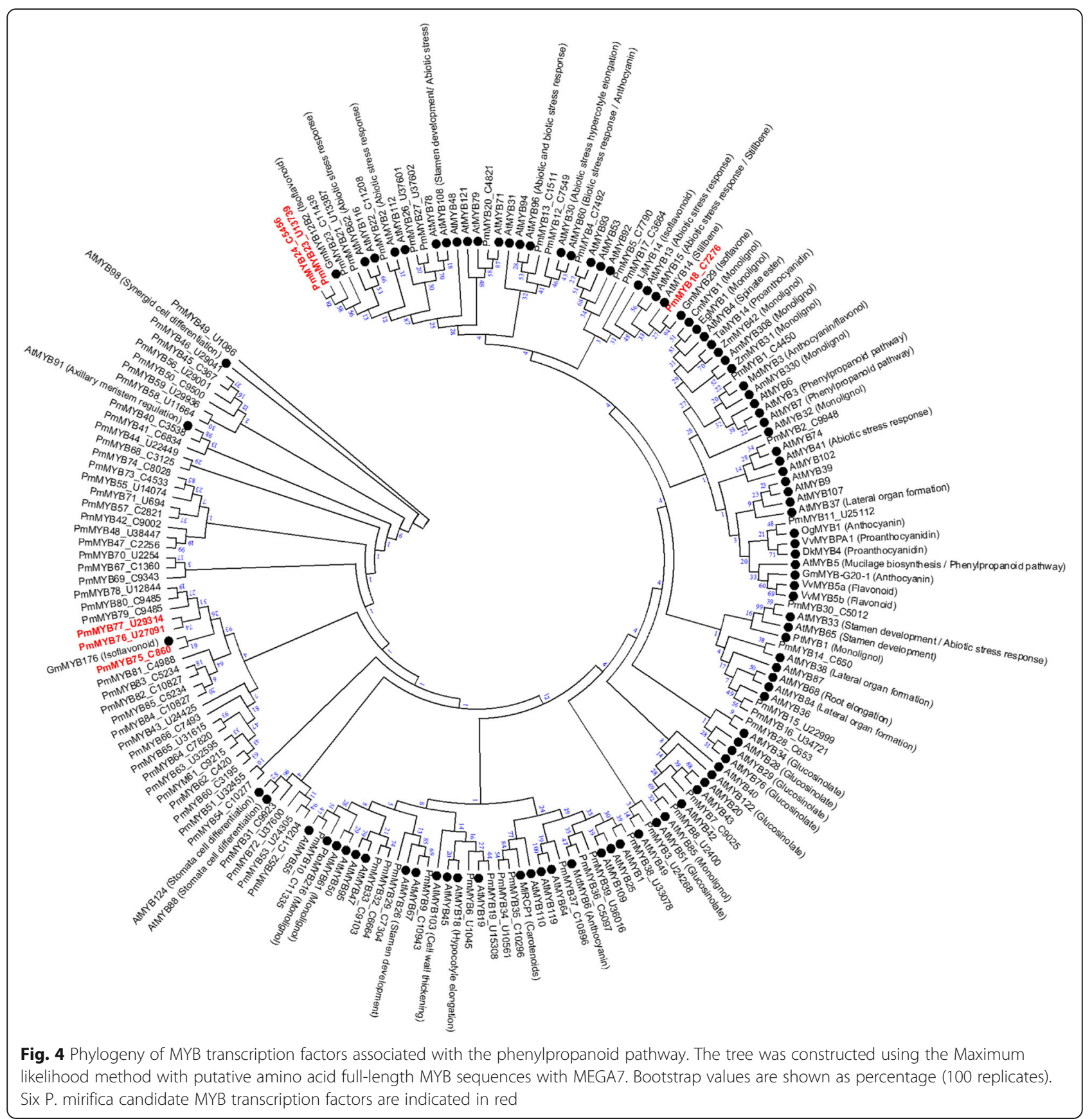

activator of flavonoid biosynthesis [29]. This led to accumulation of genistein and genistin (Additional file 6: Figure S6). The demonstrated in planta function of PmIFS suggests that our transcriptome libraries were suitable for identifying other genes involved in miroestrol biosynthesis. Although in a previous study, a biosynthetic pathway of miroestrol was tentatively suggested [15], the genes required for miroestrol biosynthesis remained unknown so far. Here, the biosynthetic pathway of miroestrol was reconsidered and the most plausible mechanism was proposed (Fig. 1). Seven putative genes encoding three biosynthetic enzymes involved in our proposed miroestrol biosynthetic pathway were also identified in our transcriptome data. Accumulation of deoxymiroestrol and miroestrol was reported to be higher in tuberous cortices than in cortex-excised tubers of $P$. mirifica [10]. Similarly, we detected deoxymiroestrol specifically in the tuberous cortices of $P$. mirifica; miroestrol, however, was not detected. Miroestrol can be non-enzymatically converted from deoxymiroestrol at high temperatures or in acidic or alkaline solutions during storage and extraction $[38,39]$. In our experiment, $P$. 

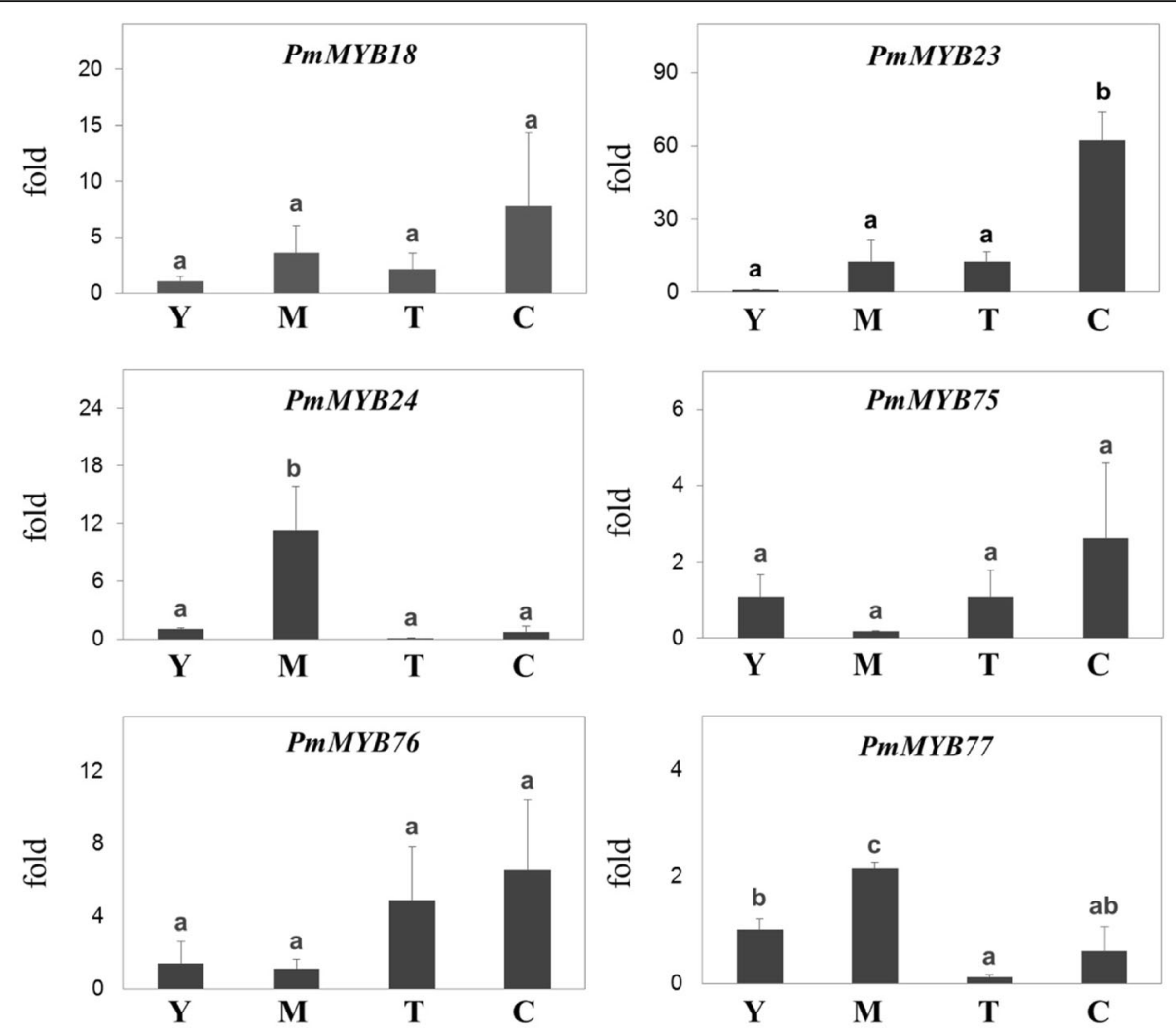

Fig. 5 Relative expression of the candidate MYB TFs involved in isoflavone biosynthesis across four tissues of $P$. mirifica by qRT-PCR. Tissue abbreviations: Y; young leaves, M; mature leaves, T; cortex-excised tubers, C; tuberous cortices. Significantly different expression levels of putative genes (ANOVA, post-hoc Duncan test, $P<0.05$ ) have different lowercase letters

mirifica tissues were harvested and rapidly frozen in liquid nitrogen, which would prevent conversion of deoxymiroestrol to miroestrol. In addition, the proposed intermediates 2 '-hydroxydaidzein and 3'-hydroxydaidzein were not detected in any tissue. We hypothesized that an organized multienzyme cluster, known as metabolon, may be involved in miroestrol biosynthesis and be responsible for the absence of those cryptic biosynthetic intermediates. In fact, an increasing number of studies describe the organization of biosynthetic pathways as metabolons. Particularly, one recent study showed efficient and specific substrate transformation in a metabolon complex between IFS and chalcone reductase in soybean plants [40]. In addition, the formation of a metabolon channeling substrate towards a product without the leakage of labile intermediates has been shown [41].

Regarding putative genes involved in miroestrol biosynthesis in P. mirifica, we found that CYP81E, IFR, and $P T$ genes were highly expressed in mature leaves, which were not the accumulation sites of miroestrol and deoxymiroestrol. Perhaps those intermediates or deoxymiroestrol are initially synthesized in mature leaves and are converted to a soluble form of glycoside that is readily transported across organs and is then stored predominantly in tuberous cortices. In fact, a glycosylated form of miroestrol has been identified in the $P$. mirifica tuber [42]. Examples of such transports are also known in tobacco, where nicotine is transported from the roots where it is biosynthesized site to the leaves where it is accumulated. Secondary metabolites are translocated between plant cells through secondary transporters and are then accumulated in the appropriate tissues or organ [43]. However, the transport of intermediates of deoxymiroestrol is still unclear and should be further investigated. Nevertheless, comprehensive functional characterization of biosynthetic genes and isotopic labeling of any intermediates is required to test this hypothesis. Alternatively, we do not exclude the possibility that deoxymiroestrol and intermediates could also be biosynthesized in the tuberous cortex, as the corresponding biosynthetic gene expression was lower there than in mature leaves. These lower expression levels of putative miroestrol biosynthetic genes could be partially responsible for the low accumulation levels of deoxymiroestrol and miroestrol. In addition, we found considerably high amounts of puerarin in tuberous cortices. 
Since puerarin, deoxymiroestrol, and miroestrol share an early biosynthetic pathway (Fig. 1), daidzein may be primarily diverted into the puerarin biosynthetic pathway, producing lower daidzein levels for miroestrol biosynthesis. Moreover, we phylogenetically identified a total of five putative genes annotated as $C$-glycosyltransferase, which plays a key role in producing 8-C-glycosylation [44] in the puerarin biosynthetic pathway. One of these putative genes, CL7002, showed $92.32 \%$ similarity to previously identified P. lobata C-glycosyltransferase (CGT43). These putative genes were highly expressed in tuberous cortices as compared to the other tissues (Additional file 5: Figure S5). Additionally, several glycosyltransferases have been shown to form a metabolon complex for facilitating efficient production of bioactive compounds [45-47]. These observations could contribute predominantly to daidzein utilization for producing puerarin. Suppression of PmCGT may be an alternative strategy to enhance miroestrol production in tubers.

A different strategy to enhance the production of isoflavonoids and their valuable derivatives is to manipulate transcription factor regulation. In this study, we identified MYB TF, which generally acts either as a transcriptional activator or repressor for various groups of plant secondary metabolites, including isoflavonoids. Amino acid alignments and expression patterns are informative for determining the functions of individual MYB TFs. Six candidate MYBs (indicated in red in the phylogenetic tree in Fig. 4) are proposed due to their phylogenetic clustering with characterized MYB TFs that are involved in the regulation of isoflavonoid biosynthetic genes. Interestingly, four of these transcription factor genes were highly expressed in the tuberous cortex, which showed the highest accumulation of isoflavones (Fig. 5). Moreover, two of these transcription factors, PmMYB18 and PmMYB75, shared one branch with those MYBs reported as activators for transcription of chalcone reductase and IFS and increased isoflavonoid production [30, 34]. These two MYBs are thus important candidates for further functional characterization, and PmMYB TFs may be associated with the regulation of isoflavonoid biosynthesis and miroestrol production.

\section{Conclusions}

We identified several candidate genes encoding key enzymes or transcription factors involved in the biosynthesis of isoflavonoid and miroestrol. Integrative analyses of transcriptomics and metabolomics indicated the complexity of gene expression and metabolite profiles across tissues, suggesting that cortical tuber tissue is a major site of isoflavonoid biosynthesis. Further molecular and biochemical studies on candidate genes involved in miroestrol biosynthesis are required to identify the functions of these candidate genes.

\section{Methods}

Plant material, chemicals, and reagents

Leaves and tubers of an approximately two-year-old $P$. mirifica cultivar SARDI190 (Fig. 2a-d) were obtained from a $P$. mirifica farm at Kasetsart University, Kamphaeng Saen Campus, Nakhonpathom, Thailand (14. $1^{\prime}$ $\left.\mathrm{N}, 99^{\circ} 58^{\prime} \mathrm{E}\right)$. The species was confirmed to be $P$. mirifica by comparison with the voucher specimens no. BCU010250 and BCU011045 kept at the Professor Kasin Suvatabhandhu Herbarium, Department of Botany, Faculty of Science, Chulalongkorn University, Thailand. Research on P. mirifica has been approved by the Department of Thai Traditional and Alternative Medicine, Ministry of Public Health, Thailand (DTAM1-2/2561). Seeds of $N$. benthamiana were germinated on sterilized soil in a culture room at $25^{\circ} \mathrm{C}$ under a $16 / 8 \mathrm{~h}$ light/dark cycle. After germinating for five days, $N$. benthamiana seedlings were watered twice per week until harvest. Standard compounds of 2'-hydroxydaidzein [22] and 3'hydroxydaidzein [22] were obtained from Dr. Tomoyoshi Akashi, Nihon University, Japan. Daidzein and genistein were purchased from CALBIOCHEM (Merck, Germany), and daidzin, genistin, and puerarin were purchased from Sigma-Aldrich (USA). All other chemicals were commercially available products of analytical-reagent grade.

\section{Construction of de novo transcriptome assembly from the combined RNA of $P$. mirifica using Illumina Hiseq 2000}

Total RNA was isolated from young leaves, mature leaves, cortex-excised tubers, and tuberous cortices using the Rneasy Plant Mini Kit (Qiagen, USA), and oncolumn DNA was removed using the Rnase-free Dnase Set (Qiagen, USA). RNA quality was examined by agarose gel electrophoresis, and quantity was measured using a biospectrometer (Eppendorf, Germany). Aliquots of high-quality RNA of all four tissue types were pooled and subjected to Illumina Hiseq 2000 sequencing at BGI, Hongkong. Raw reads were filtered using an inhouse software of BGI, filter fq, to produce clean pairedend reads and unpaired reads. Contigs and unigenes were then assembled using Trinity software [48]. We used a BUSCO (Benchmarking Single-Copy Universal Orthologs) version 3.0.2 analysis against the eudicotyledons_odb10 dataset $[49,50]$ to assess completeness. Raw transcriptome data were deposited in the NCBI sequence read archive (accession number: SRR6917866).

\section{Analysis of gene expression levels across the four tissues} of $P$. mirifica generated using Illumina NextSeq500

Total RNA was extracted from each tissue type using three biological replicates. Based on spectrophotometry, high-quality RNA aliquots of the replicates were pooled per tissue type at equal quantities. The four RNA pools were then subjected to pair-end sequencing using an 
Illumina NextSeq500 platform. Contigs were assembled using Trinity software [48]. To estimate gene expression levels, we mapped all reads in fastq format to the contigs and calculated the reads per kilobase of the transcript per million mapped reads values. A non-parametric approach for identifying DEGs was used to produce four independent pairwise sample comparisons with the softawre NOISeq-sim [51]. Raw transcriptome data were deposited in the NCBI sequence read archive (accession numbers SRR10177497, SRR10177499, SRR10177500, and SRR10177498 for sequences produced from young leaves, mature leaves, cortex-excised tubers, and tuberous cortices, respectively.)

\section{Functional annotation of assembled sequences}

The assembled sequences were first compared against the NCBI non-redundant protein database (https://www. ncbi.nlm.nih.gov/refseq/), Swiss-Prot (https://www.ebi. ac.uk/uniprot), Kyoto Encyclopedia of Genes and Genomes (KEGG; http://www.kegg.jp/kegg/), and clusters of orthologous groups (COG; $e$-value $<1 E-5$; https:// www.ncbi.nlm.nih.gov/COG/) by BLASTx, and nucleotide database NT (e-value <1E-5; https://www.ncbi.nlm. nih.gov/nucleotide/) using BLASTn. Then, the numbers of unigenes annotated from each database were counted. Unigenes were mapped to the COG database, potential functions were predicted, and statistical analyses were performed. BLAST2GO software v2.5.0 [52] was used to assign GO categories based on BLAST results, using default settings. All unigene GO functional categories and distribution of gene functions in different species were visualized using WEGO software [53].

\section{Phylogenetic analyses}

All putative genes were translated to amino acid sequences using the Expasy translate tool (http://web. expasy.org/translate/). All full-length sequences of putative genes involved in isoflavonoid and chromene synthesis were aligned using BioEdit software (http://www. mbio.ncsu.edu/BioEdit/bioedit.html). We applied a maximum likelihood method with 100 bootstrap replicates using MEGA7 software [54] to produce phylogenetic trees.

\section{Assessment of candidate gene expression using qRT-PCR}

Transcription levels of candidate genes were assessed using qRT-PCR. Total RNA isolated from the four tissue types was used (Fig. 2a-d) to synthesize single-stranded cDNA using the RevertAid First Strand cDNA Synthesis Kit (Thermo Fisher, USA). The qRT-PCR was performed using equal amounts of template DNA in a CFX96 TouchTM Real-Time PCR Detection system with iTaq Universal SYBR Green Supermix (Biorad, USA) and under the following conditions: $95^{\circ} \mathrm{C}$ for $8 \mathrm{~min}$ followed by 40 cycles of $95^{\circ} \mathrm{C}$ for $10 \mathrm{~s}, 60^{\circ} \mathrm{C}$ for $15 \mathrm{~s}$, and $72{ }^{\circ} \mathrm{C}$ for $30 \mathrm{~s}$. The specific primers used for amplification of candidate gene sequences are shown in Additional file 10: Table S4. The $P$. mirifica unigene encoding EF1 $\alpha$ was amplified as an internal control. Each reaction was performed using three biological replicates. The relative expression ratios of the candidate genes expressed in leaves and tuberous roots were calculated and normalized to a reference gene $(E F 1 \alpha)$ transcript level in the same sample [55].

\section{Metabolite extraction from $P$. mirifica samples}

$P$. mirifica samples were frozen, ground to a fine powder, and lyophilized overnight. Thirty milligrams of dried powder were dissolved in $500 \mu \mathrm{L}$ methanol (85\%) to extract metabolites under vigorous shaking using a Mixer Mill MM400 (Retsch, Germany) at $25 \mathrm{~Hz}$ for $7 \mathrm{~min}$. Crude metabolite extracts were filtered through an Acrodisc $^{\circ}$ Syringe Filter with a $0.2-\mu \mathrm{m}$ Supor ${ }^{\circ}$ membrane (Pall, USA) and stored at $-20^{\circ} \mathrm{C}$ until analysis.

\section{Phytoestrogen profiling by HPLC quadrupole time of flight mass spectrometry (HPLC-QTOF-MS/MS)}

The HPLC-QTOF-MS/MS analysis was performed using an Agilent HPLC 1260 series device coupled with a QTOF 6540 UHD Accurate-Mass system (Agilent Technologies, Germany). The separation of the sample solution was performed on a Luna C18 column (2) $150 \times 4.6$ $\mathrm{mm}, 5 \mu \mathrm{m}$ (Phenomenex, USA). The solvent flow rate was $0.5 \mathrm{~mL} / \mathrm{min}$, with $5 \mu \mathrm{L}$ of the sample solution being injected into the LC system. The binary gradient elution system was composed of water as solvent A and acetonitrile as solvent $\mathrm{B}$, with both solvents containing $0.1 \%$ formic acid (v/v). The linear gradient elution was 5-95\% for solvent $\mathrm{B}$ at $35 \mathrm{~min}$ with a post-run for $5 \mathrm{~min}$. The column temperature was set at $40^{\circ} \mathrm{C}$. The conditions for the negative ESI source were as follows: drying gas $\left(\mathrm{N}_{2}\right)$ flow rate $10 \mathrm{~L} / \mathrm{min}$, drying gas temperature $350{ }^{\circ} \mathrm{C}$, nebulizer $30 \mathrm{psig}$, the fragmentor set to $100 \mathrm{~V}$, capillary voltage $3500 \mathrm{~V}$, and scan spectra from $\mathrm{m} / z$ 100-1500. The auto MS/MS for the fragmentation was set at collision energies of 10,20 , and $40 \mathrm{~V}$. All data analyses were performed using Agilent MassHunter Qualitative Analysis Software B06.0 (Agilent Technologies, USA).

\section{Molecular cloning and transient expression of PmIFS and AtMYB12 in N. benthamiana}

The unigene encoding isoflavone synthase (PmIFS; NCBI sequence accession number MK524721) was amplified from $P$. mirifica $\mathrm{cDNA}$ using the following specific primers: forward 5' -ATGTTGCTGGAACTTGCAATTG$3^{\prime}$ and reverse 5' -TCAAGAAGGAGGTTTAGATGC-3'. For Arabidopsis MYB12 (AtMYB12; accession number NM130314), the full-length gene sequence was amplified 
from Arabidopsis cDNA using the following gene-specific primers: forward 5' - ATGGGAAGAGCGCCATGT-3' and reverse 5' - TCATGACAGAAGCCAAGCG-3'. The PCR was performed using $50 \mu \mathrm{L}$ reaction volumes and a Phusion ${ }^{\bullet}$ HF high-fidelity DNA polymerase (Thermo Fisher Scientific, Finland) with the following thermocycling protocol: $98^{\circ} \mathrm{C}$ for $1 \mathrm{~min}$ followed by 35 cycles of $98^{\circ} \mathrm{C}$ for $10 \mathrm{~s}, 60^{\circ} \mathrm{C}$ for $30 \mathrm{~s}, 72^{\circ} \mathrm{C}$ for $1 \mathrm{~min}$, and a final extension step at $72^{\circ} \mathrm{C}$ for $5 \mathrm{~min}$. PCR products were visualized by agarose gel electrophoresis. Amplicons of target size were ligated into a pJET1.2 vector (Fermentas, USA) for sequencing. The genes were then sub-cloned into pDONOR207 and pEAQ-HT-DEST1 [56] vectors, respectively, using a gateway cloning system according to manufacturer's instructions (Invitrogen, USA). The recombinant pEAQ-HT-DEST1 vectors harboring PmIFS and pEAQ-HT-DEST1 vectors harboring AtMYB12 were electro-transformed into Agrobacterium tumefaciens LBA4404. Positive clones were subjected to colony PCR. A single colony of each transformant was grown overnight (at $28^{\circ} \mathrm{C}$ and under rotation at $250 \mathrm{rpm}$ ) in $5 \mathrm{~mL} \mathrm{YEB}$ medium supplemented with $100 \mu \mathrm{g} / \mathrm{mL}$ rifampicin, $50 \mu \mathrm{g} / \mathrm{mL}$ kanamycin, and $100 \mu \mathrm{g} / \mathrm{mL}$ streptomycin. The cultures were then washed three times using sterilized distilled water. The resulting pellets were placed in a resuspension solution $\left(10 \mathrm{mM} \mathrm{MgCl}_{2}, 10\right.$ $\mathrm{mM}$ MES-K, and $100 \mu \mathrm{M}$ acetosyringone, at $\mathrm{pH}$ 5.6). The Agrobacterium solution was adjusted to an $\mathrm{A}_{600}$ of approximately 0.4 and placed on a bench at room temperature for $2-3 \mathrm{~h}$ before infiltration. The Agrobacterium solution was infiltrated into the third and the fourth leave (from the shoot tip) of approximately four-week old $N$. benthamiana plants. After five days, the infiltrated leaves were rapidly frozen using liquid nitrogen and were subsequently ground to a powder. The powder was immediately lyophilized and was then vacuum-stored at room temperature. Thirty milligrams of powder were dissolved in $500 \mu \mathrm{L}$ of $85 \%$ methanol, and metabolites were extracted by vigorous shaking using a Mixer Mill MM400 (Retsch, Germany) at $25 \mathrm{~Hz}$ for $7 \mathrm{~min}$. The crude extracts were filtered through an Acrodisc ${ }^{\circ}$ Syringe Filter with a $0.2 \mu \mathrm{m}$ Supor $^{\circledR}$ membrane (Pall, USA).

\section{Supplementary information}

Supplementary information accompanies this paper at https://doi.org/10. 1186/s12870-019-2205-0.

Additional file 1: Figure S1. Whole plant of the approximately 3-yearold P. mirifica. (bar $=5 \mathrm{~cm}$ )

Additional file 2: Figure S2. Clusters of orthologous groups (COG) functional classification for all assembled unigenes in $P$. mirifica. The vertical coordinates are function classes of COG, and the horizontal coordinates are numbers of unigenes.

Additional file 3: Figure S3. Gene ontology (GO) annotation for all assembled unigenes in P. mirifica. The 56 subcategories are affiliated to three main domains: biological process, cellular component, and molecular function. The GO categories were created using WEGO software.

Additional file 4: Figure S4. KEGG pathway enrichment analysis of assembled unigenes in P. mirifica. a The Number of unigenes in 19 subcategories of metabolic pathway category. $\mathbf{b}$ The 21 sub-categories of metabolism of terpenoids and polyketides, and other secondary metabolites.

Additional file 5: Figure S5. Differential expressed genes (DEGs) predicted as UDP-glycosyltransferases that might be involved in isoflavone biosynthetic pathway across the four tissues of $P$. mirifica.

Additional file 6: Figure S6. Ion extract chromatogram of the annotated genistein $([\mathrm{M}-\mathrm{H}]=269, \mathrm{RT}=18.78)$ of methanolic extracts of GFP transiently overexpressed in N. benthamiana (a), P. mirifica Isoflavone synthase (PmIFS) transiently expressed in $N$. benthamiana $(\mathbf{b})$, and $P$. mirifica Isoflavone synthase (PmIFS) and Arabidopsis MYB12 transcription factor transiently co-expressed in $\mathrm{N}$. benthamiana (c), as detected by HPLC-QTOF-MS/MS in negative mode.

Additional file 7: Table S1. Summary of the $P$. mirifica transcriptome assembly.

Additional file 8: Table S2. Annotation statistics.

Additional file 9: Table S3. Nucleotide sequences and expression profiles across the four tissues of biosynthetic genes and transcription factors involved in isoflavone and miroestrol biosyntheses.

Additional file 10: Table S4. DNA primer list for $q R T-P C R$ validation.

\section{Abbreviations}

4CL: 4-coumarate CoA ligase; BUSCO: Benchmarking Single-Copy Universal Orthologs; C: Tuberous cortices; C4H: Cinnamate 4-hydroxylase; CGT: Cglycosyltransferase; $\mathrm{CHI}$ : Chalcone isomerase; CHR: Chalcone reductase; CHS: Chalcone synthase; COG: Clusters of orthologous groups;

CYP81E: Cytochrome P450 subfamily 81E; DEG: Differential expressed gene; DMAPP: Dimethylallyl pyrophosphate; EF1a: Elongation factor 1 alpha; GFP: Green fluorescent protein; GO: Gene ontology; HID: 2hydroxyisoflavanone dehydratase; HPLC-QTOF-MS/MS: High performance liquid chromatography quadrupole time of flight tandem mass spectrometry; IFR: Isoflavone reductase; IFS: Isoflavone synthase; KEGG: Kyoto encyclopedia of genes and genomes; M: Mature leaves; MYB TF: v-myb myeloblastosis viral oncogene homolog transcription factor; nr : NCBI non-redundant protein database; PAL: Phenylalanine ammonia lyase; PT: Prenyltransferase; RPKM: Read Per Kilobase of Transcript Per Million Mapped Reads; SRA: Sequence read archive; T: Cortex-excised tubers; Y: Young leaves

\section{Acknowledgements}

We would like to thank Dr. Tomoyoshi Akashi (Nihon University, Japan) for providing standard compounds. We extend our gratitude to Dr. George Lomonossoff (John Innes Centre, UK) and Plant Bioscience Limited (UK) for supplying pEAQ vector. HS thanks Frédéric Tournay at the Botanical Garden of the University of Strasbourg for horticultural work and taking care of the plants.

\section{Authors' contributions}

NS designed and performed the experiments, including data analysis, and wrote the manuscript. KT, PP, JZ, HS, KK, and SS analyzed the data. SW contributed materials and analysis tools. SS, SV, HS, MY, KS, and WD conceived and designed the study and revised the manuscript. All authors read and approved the manuscript.

\section{Funding}

The authors express their gratitude to Chulalongkorn University for providing research funds (CU-56-508-HR and GRU 6101133003-1). N.S. received a student fellowship from the 100th Anniversary of Chulalongkorn University Fund for Doctoral Scholarship, an Overseas Research Experience Scholarship for Graduate Students from the Graduate School, Chulalongkorn University, and the FY2018 Thesis Grant for Ph.D. Students, National Research Council of Thailand. This research was also partly supported by the Research Unit for Natural Product Biotechnology, Chulalongkorn University, the Chulalongkorn Academic Advancement into Its second Century Project, and the Franco-Thai 
Cooperation Program in Higher Education and Research together with the French PHC program SIAM2014-2015. HS and WD thank the French Ministry of Foreign Affairs, Campus France, and the French embassy in Bangkok for supporting the BIOACTIVE NATURAL PRODUCTS FROM PLANTS project (project $n^{\circ} 31827 T$ J). The funding bodies were not involved in the design of the study and collection, data analyses, data interpretation, and writing of the manuscript.

\section{Availability of data and materials}

The datasets used and/or analyzed during the current study are available from the corresponding author on reasonable request.

\section{Ethics approval and consent to participate}

Not applicable.

\section{Consent for publication}

Not applicable.

\section{Competing interests}

The authors declare that they have no competing interests.

\section{Author details}

'Department of Biochemistry, Faculty of Science, Chulalongkorn University, Bangkok, Thailand. ${ }^{2}$ Omics Sciences and Bioinformatics Center, Chulalongkorn University, Bangkok, Thailand. ${ }^{3}$ Graduate Program in Pharmaceutical Science and Technology, Faculty of Pharmaceutical Sciences, Chulalongkorn University, Bangkok, Thailand. ${ }^{4}$ Institut de Biologie Moléculaire des Plantes, CNRS, Université de Strasbourg, Strasbourg, France. ${ }^{5}$ Department of Animal Science, Faculty of Agriculture at Kamphaeng Saen, Kasetsart University, Nakhon Pathom, Thailand. '́Laboratory of Molecular Biology and Biotechnology, Graduate School of Pharmaceutical Sciences, Chiba University, Chiba, Japan. ${ }^{7}$ Natural Product Biotechnology Research Unit, Department of Pharmacognosy and Pharmaceutical Botany, Faculty of Pharmaceutical Sciences, Chulalongkorn University, Bangkok, Thailand.

Received: 28 June 2019 Accepted: 16 December 2019

Published online: 26 December 2019

\section{References}

1. Malaivijitnond S. Medical applications of phytoestrogens from the Thai herb Pueraria mirifica. Front Med. 2012;6(1):8-21.

2. Ososki AL, Kennelly EJ. Phytoestrogens: a review of the present state of research. Phytother Res. 2003;17(8):845-69.

3. Ingham JL, Tahara S, Dziedzic SZ. A chemical investigation of Pueraria mirifica roots. Z Naturforsch C. 1986;41(4):403-8.

4. Cherdshewasart W, Subtang S, Dahlan W. Major isoflavonoid contents of the phytoestrogen rich-herb Pueraria mirifica in comparison with Pueraria lobata. J Pharm Biomed Anal. 2007:43(2):428-34.

5. Cherdshewasart W, Sriwatcharakul S. Major isoflavonoid contents of the 1year-cultivated phytoestrogen-rich herb, Pueraria mirifica. Biosci Biotechnol Biochem. 2007;71(10):2527-33.

6. Veitch NC. Isoflavonoids of the Leguminosae. Nat Prod Rep. 2013;30(7):988-1027.

7. Ingham JL, Tahara S, Dziedzic SZ. Coumestans from the roots of Pueraria mirifica. Z Naturforsch C. 1988:43(1-2):5-10.

8. Costa M, Dias TA, Brito A, Proença F. Biological importance of structurally diversified chromenes. Eur J Med Chem. 2016;123:487-507.

9. Jones HE, Pope GS. A method for the isolation of miroestrol from Pueraria mirifica. J Endocrinol. 1961;22(3):303-12.

10. Yusakul G, Putalun W, Udomsin O, Juengwatanatrakul T, Chaichantipyuth C. Comparative analysis of the chemical constituents of two varieties of Pueraria candollei. Fitoterapia. 2011;82(2):203-7.

11. Cain JC. Miroestrol: an oestrogen from the plant Pueraria mirifica. Nature. 1960;188:774-7.

12. Dixon RA, Achnine L, Kota P, Liu C-J, Reddy MSS, Wang L. The phenylpropanoid pathway and plant defence-a genomics perspective. Mol Plant Pathol. 2002;3(5):371-90.

13. Wiriyaampaiwong $P$, Thanonkeo S, Thanonkeo P. Molecular characterization of isoflavone synthase gene from Pueraria candollei var. mirifica. Afr J Agric Res. 2012;7(32):4489-98.
14. Wiriyaampaiwong P, Thanonkeo S, Thanonkeo P. Cloning and characterization of chalcone synthase gene from Pueraria candollei var. mirifica. J Med Plant Res. 2012;6(42):5469-79.

15. Udomsuk L, Juengwattanatrakul T, Jarukamjorn K, Putalun W. Increased miroestrol, deoxymiroestrol and isoflavonoid accumulation in callus and cell suspension cultures of Pueraria candollei var. mirifica. Acta Physiol Plant. 2011;34(3):1093-100

16. Liu J, Osbourn A, Ma P. MYB transcription factors as regulators of phenylpropanoid metabolism in plants. Mol Plant. 2015;8(5):689-708.

17. Han R, Takahashi H, Nakamura M, Yoshimoto N, Suzuki H, Shibata D, Yamazaki M, Saito K. Transcriptomic landscape of Pueraria lobata demonstrates potential for phytochemical study. Front Plant Sci. 2015;6:426.

18. Wang X, Li S, Li J, Li C, Zhang Y. De novo transcriptome sequencing in Pueraria lobata to identify putative genes involved in isoflavones biosynthesis. Plant Cell Rep. 2015:34(5):733-43.

19. Pang $T$, Ye $C-Y, X i a X$, Yin W. De novo sequencing and transcriptome analysis of the desert shrub, Ammopiptanthus mongolicus, during cold acclimation using Illumina/Solexa. BMC Genomics. 2013;14(1):488.

20. Huang J, Guo X, Hao X, Zhang W, Chen S, Huang R, Gresshoff PM, Zheng Y. De novo sequencing and characterization of seed transcriptome of the tree legume Millettia pinnata for gene discovery and SSR marker development. Mol Breed. 2016;36(6):75.

21. Liu Z, Chen T, Ma L, Zhao Z, Zhao PX, Nan Z, Wang Y. Global Transcriptome sequencing using the Illumina platform and the development of EST-SSR markers in Autotetraploid alfalfa. PLoS One. 2013;8(12):e83549.

22. Akashi T, Aoki T. Ayabe S-i: CYP81E1, a cytochrome P450 CDNA of licorice (Glycyrrhiza echinata L.), encodes isoflavone 2'-hydroxylase. Biochem Biophys Res Commun. 1998:251(1):67-70.

23. Liu CJ, Huhman D, Sumner LW, Dixon RA. Regiospecific hydroxylation of isoflavones by cytochrome P450 81E enzymes from Medicago truncatula. Plant J. 2003;36(4):471-84.

24. Overkamp S, Hein F, Barz W. Cloning and characterization of eight cytochrome P450 cDNAs from chickpea (Cicer arietinum L.) cell suspension cultures. Plant Sci. 2000;155(1):101-8.

25. Shimada N, Akashi T, Aoki T. Ayabe S-i: induction of isoflavonoid pathway in the model legume Lotus japonicus: molecular characterization of enzymes involved in phytoalexin biosynthesis. Plant Sci. 2000;160(1):37-47.

26. Guijas C, Montenegro-Burke JR, Domingo-Almenara X, Palermo A, Warth B, Hermann G, Koellensperger G, Huan T, Uritboonthai W, Aisporna AE, et al. METLIN: a technology platform for identifying Knowns and unknowns. Anal Chem. 2018:90(5):3156-64

27. Lee JH, Kim JY, Cho S-H, Jeong JH, Cho S, Park HJ, Baek SY. Determination of miroestrol and isomiroestrol from Pueraria mirifica (white Kwao Krua) in dietary supplements by LC-MS-MS and LC-Q-Orbitrap/MS. J Chromatogr Sci. 2017:55(3):214-21.

28. Sainsbury F, Lomonossoff GP. Transient expressions of synthetic biology in plants. Curr Opin Plant Biol. 2014;19:1-7.

29. Pandey A, Misra P, Khan Mohd P, Swarnkar G, Tewari Mahesh C, Bhambhani S, Trivedi R, Chattopadhyay N, Trivedi Prabodh K. Co-expression of Arabidopsis transcription factor, AtMYB12, and soybean isoflavone synthase, GmIFS1, genes in tobacco leads to enhanced biosynthesis of isoflavones and flavonols resulting in osteoprotective activity. Plant Biotechnol J. 2013; 12(1):69-80.

30. Chu S, Wang J, Zhu Y, Liu S, Zhou X, Zhang H. Wang C-e, Yang W, Tian Z, Cheng $\mathrm{H}$ et al: an R2R3-type MYB transcription factor, GmMYB29, regulates isoflavone biosynthesis in soybean. PLoS Genet. 2017;13(5):e1006770.

31. Yi J, Derynck MR, Chen L, Dhaubhadel S. Differential expression of CHS7 and CHS8 genes in soybean. Planta. 2010;231(3):741-53.

32. Li X-W, Li J-W, Zhai Y, Zhao Y, Zhao X, Zhang H-J, Su L-T, Wang Y, Wang QY. A R2R3-MYB transcription factor, GmMYB12B2, affects the expression levels of flavonoid biosynthesis genes encoding key enzymes in transgenic Arabidopsis plants. Gene. 2013;532(1):72-9.

33. Yi J, Derynck MR, Li X, Telmer P, Marsolais F, Dhaubhadel S. A singlerepeat MYB transcription factor, GmMYB176, regulates CHS8 gene expression and affects isoflavonoid biosynthesis in soybean. Plant J. 2010:62(6):1019-34

34. Yu O, Shi J, Hession AO, Maxwell CA, McGonigle B, Odell JT. Metabolic engineering to increase isoflavone biosynthesis in soybean seed. Phytochemistry. 2003;63(7):753-63.

35. Reuter Jason A, Spacek DV, Snyder Michael P. High-throughput sequencing technologies. Mol Cell. 2015;58(4):586-97. 
36. Serres-Giardi L, Belkhir K, David J, Glémin S. Patterns and evolution of nucleotide landscapes in seed plants. Plant Cell. 2012;24(4):1379-97.

37. Tian A-G, Wang J, Cui P, Han Y-J, X U H, Cong L-J, Huang X-G, Wang X-L, Jiao YZ, Wang B-J, et al. Characterization of soybean genomic features by analysis of its expressed sequence tags. Theor Appl Genet. 2004;108(5):903-13.

38. Chansakaow S, Ishikawa T, Sekine K, Okada M, Higuchi Y, Kudo M, Chaichantipyuth C. Isoflavonoids from Pueraria mirifica and their estrogenic activity. Planta Med. 2000;66(06):572-5.

39. Udomsin O, Juengwatanatrakul T, Yusakul G, Putalun W. Chromene stability: the most potent estrogenic compounds in white Kwao Krua (Pueraria candollei var mirifica) crude extract. J Funct Foods. 2015;19:269-77.

40. Mameda R, Waki T, Kawai Y, Takahashi S, Nakayama T. Involvement of chalcone reductase in the soybean isoflavone metabolon: identification of GmCHR5, which interacts with 2-hydroxyisoflavanone synthase. Plant J. 2018:96(1):56-74

41. Laursen T, Møller BL, Bassard J-E. Plasticity of specialized metabolism as mediated by dynamic metabolons. Trends Plant Sci. 2015;20(1):20-32.

42. Bang M-H, Lee D-G, Baek Y-S, Cho J-G, Han M-W, Choi K-S, Chung D-K, Ko S-K, Oh C-H, Cho S-Y, et al. A new miroestrol glycoside from the roots of Pueraria mirifica. Chem Nat Compounds. 2013:49:443.

43. Yazaki K, Sugiyama A, Morita M, Shitan N. Secondary transport as an efficient membrane transport mechanism for plant secondary metabolites. Phytochem Rev. 2008;7(3):513-24

44. Wang X, Li C, Zhou C, Li J, Zhang Y. Molecular characterization of the Cglucosylation for puerarin biosynthesis in Pueraria lobata. Plant J. 2017;90(3): 535-46.

45. Nielsen KA, Tattersall DB, Jones PR, Møller BL. Metabolon formation in dhurrin biosynthesis. Phytochemistry. 2008:69(1):88-98.

46. Jørgensen K, Rasmussen AV, Morant M, Nielsen AH, Bjarnholt N, Zagrobelny M, Bak S, Møller BL. Metabolon formation and metabolic channeling in the biosynthesis of plant natural products. Curr Opin Plant Biol. 2005;8(3):280-91.

47. Laursen T, Borch J, Knudsen C, Bavishi K, Torta F, Martens HJ, Silvestro D, Hatzakis NS, Wenk MR, Dafforn TR, et al. Characterization of a dynamic metabolon producing the defense compound dhurrin in sorghum. Science. 2016;354(6314):890.

48. Haas BJ, Papanicolaou A, Yassour M, Grabherr M, Blood PD, Bowden J, Couger MB, Eccles D, Li B, Lieber M, et al. De novo transcript sequence reconstruction from RNA-seq using the trinity platform for reference generation and analysis. Nat Protoc. 2013:8(8):1494-512.

49. Simão FA, Waterhouse RM, loannidis P, Kriventseva EV, Zdobnov EM. BUSCO: assessing genome assembly and annotation completeness with single-copy orthologs. Bioinformatics. 2015;31(19):3210-2.

50. Waterhouse RM, Seppey M, Simão FA, Manni M, loannidis P, Klioutchnikov G, Kriventseva EV, Zdobnov EM. BUSCO applications from quality assessments to gene prediction and Phylogenomics. Mol Biol Evol. 2017 35(3):543-8.

51. Tarazona S, García-Alcalde F, Dopazo J, Ferrer A, Conesa A. Differential expression in RNA-seq: a matter of depth. Genome Res. 2011;21(12):2213-23.

52. Götz S, García-Gómez JM, Terol J, Williams TD, Nagaraj SH, Nueda MJ, Robles M, Talón M, Dopazo J, Conesa A. High-throughput functional annotation and data mining with the Blast2GO suite. Nucleic Acids Res. 2008:36(10):3420-35.

53. Ye J, Fang L, Zheng H, Zhang Y, Chen J, Zhang Z, Wang J, Li S, Li R, Bolund $\mathrm{L}$, et al. WEGO: a web tool for plotting GO annotations. Nucleic Acids Res. 2006:34:W293-7.

54. Kumar S, Stecher G, Tamura K. MEGA7: molecular evolutionary genetics analysis version 7.0 for bigger datasets. Mol Biol Evol. 2016;33(7):1870-4

55. Livak KJ, Schmittgen TD. Analysis of relative gene expression data using real-time quantitative PCR and the $2^{-\Delta \Delta C T}$ method. Methods. 2001;25(4): 402-8.

56. Sainsbury F, Thuenemann EC, Lomonossoff GP. pEAQ: versatile expression vectors for easy and quick transient expression of heterologous proteins in plants. Plant Biotechnol J. 2009;7(7):682-93.

\section{Publisher's Note}

Springer Nature remains neutral with regard to jurisdictional claims in published maps and institutional affiliations.

\section{Ready to submit your research? Choose BMC and benefit from}

- fast, convenient online submission

- thorough peer review by experienced researchers in your field

- rapid publication on acceptance

- support for research data, including large and complex data types

- gold Open Access which fosters wider collaboration and increased citations

- maximum visibility for your research: over $100 \mathrm{M}$ website views per year

At BMC, research is always in progress.

Learn more biomedcentral.com/submissions 